\title{
ON THE ZEROS OF VAN VLECK POLYNOMIALS
}

\author{
G. M. SHAH ${ }^{1}$
}

1. Introduction. It is known [4] that there exist $C_{n+\dot{p}-2, p-2}$ polynomials $V(x)$ of degree $(p-2)$ such that corresponding to each such $V(x)$ the differential equation

$$
\prod_{j=1}^{p}\left(x-a_{j}\right)\left[y^{\prime \prime}+\left(\sum_{j=1}^{p} \frac{\alpha_{j}}{x-a_{j}}\right) y^{\prime}\right]+V(x) y=0,
$$

where all $\alpha_{j}>0$ and $a_{1}<a_{2}<\cdots<a_{p}$, has a unique polynomial solution $S(x)$ of degree $n$. Such $S(x)$ and $V(x)$ are called Stieltjes and Van Vleck polynomials respectively [2]. It has been shown that the zeros of all such $S(x)$ and $V(x)$ lie in $\left(a_{1}, a_{p}\right)$ [1] and [5].We have proved that each $V(x)$ can have at most two zeros in any interval $\left(a_{r}, a_{r+1}\right)$, $2 \leqq r \leqq p-2$ and at most one zero in each of the intervals $\left(a_{1}, a_{2}\right)$ and $\left(a_{p-1}, a_{p}\right)[3 \mathbf{b}]$. We can, however, improve this result if we consider the distribution of the zeros of $V(x)$ in $k(k>1)$ consecutive intervals. We intend to prove the following result:

TheOREM 1. (a) Any $k(k \leqq p-1)$ consecutive intervals $\left(a_{j}, a_{j+1}\right)$, $\cdots,\left(a_{j+k-1}, a_{j+k}\right)$ contain at most $k$ and at least $(k-1)$ zeros of $V(x)$, if $j=1$ or $j=p-k$.

(b) Any $k \quad(k<p-1)$ consecutive intervals $\left(a_{j}, a_{j+1}\right), \cdots$, $\left(a_{j+k-1}, a_{j+k}\right)$ contain at most $(k+1)$ and at least $(k-1)$ zeros of $V(x)$, if $j \neq 1$ and $j \neq p-k$.

In $\$ 2$ we prove a result analogous to Rolle's theorem for the zeros of the derivative of a function with real and simple zeros. In $\$ 3$ we give the proof of Theorem 1.

2. Analogue of Rolle's theorem. As pointed out in an earlier paper [3b] we may assume that no $V(x)$ has a zero at any $a_{j}, 1 \leqq j \leqq p$, for the modification in the proof in the other case will be the obvious one. We recall from $[3 \mathbf{b}]$ the following

Lemma 1. The zeros of a Van Vleck polynomial $V(x)$ of degree $(p-2)$ and those of the corresponding $S(x)$ of degree $n$ are the zeros of the function

Received by the editors August 30, 1967.

1 The author is gratef ul to his thesis advisor, Professor M. Marden, for his guidance in this work which is part of the author's Doctoral dissertation. 


$$
G(x) \equiv \sum_{j=1}^{n-1} \frac{1}{x-x_{j}^{\prime}}+\sum_{j=1}^{p} \frac{\alpha_{j}}{x-a_{j}}
$$

and conversely, where $x_{j}{ }^{\prime}(1 \leqq j \leqq n-1)$ are the zeros of the derivative $S^{\prime}(x)$ of the $S(x)$.

Let $E$ denote the point set consisting of the zeros of $S(x)$ and those of the corresponding $V(x)$. Also, let $F$ consist of the singular points $a_{1}, a_{2}, \cdots, a_{p}$ and the zeros of the derivative $S^{\prime}(x)$ of the $S(x)$. Then, we have the following analogue of Rolle's theorem for the sets $E$ and $F$.

Theorem 2. Between any two consecutive points of $E$ lies one and only one point of $F$.

Proof. We prove first that between two consecutive points of $E$ lies one point of $F$. We consider various cases separately.

Case 1. Let the two points of $E$ under consideration be $x_{k}$ and $x_{k+1}$, both zeros of $S(x)$. Since the zeros of $S(x)$ are real and simple [3a], it follows that one zero $x_{k}^{\prime}$ of $S^{\prime}(x)$ lies between $x_{k}$ and $x_{k+1}$. $x_{k}^{\prime}$ is a point of $F$ and we have $x_{k}<x_{k}^{\prime}<x_{k+1}$.

Case 2. Let the two points of $E$ be $x_{k}$, a zero of $S(x)$ and $t_{l}$, a zero of the corresponding $V(x)$. Then, by Lemma $1, x_{k}$ and $t_{l}$ are both zeros of $G(x)$. It is easy to see that $G(x)$ has points of discontinuity at the points $a_{j}(1 \leqq j \leqq p)$ and at $x_{j}^{\prime}(1 \leqq j \leqq n-1)$. Also $G(x)$ is a continuously decreasing function of $x$ in every interval of continuity. As $G\left(x_{k}\right)=0$ and $G\left(t_{l}\right)=0$, it follows that $G(x)$ must have a discontinuity between $x_{k}$ and $t_{l}$, for otherwise it would contradict the monotonic character of $G(x)$. That point of discontinuity of $G$, being either an $a_{j}$ or a $x_{j}^{\prime}$, is a point of $F$.

Case 3. Let the two points of $E$ be $t_{k}$ and $t_{k+1}$, both zeros of $V(x)$. It can be shown as in Case 2 above, that the interval $\left(t_{k}, t_{k+1}\right)$ contains a point of discontinuity of $G$ which is a point of $F$.

We show that between any two consecutive points of $F$ is a point of $E$.

Case a. Suppose the two consecutive points of $F$ are $a_{k}, a_{k+1}$. Then $G(x)$ decreases continuously from $+\infty$ to $-\infty$ as $x$ moves from $a_{k}$ to $a_{k+1}$, because the interval $\left(a_{k}, a_{k+1}\right)$ does not contain any $x_{j}^{\prime}$. Thus $G$ vanishes precisely once in $\left(a_{k}, a_{k+1}\right)$. Since $E$ consists of the zeros of $G$, the result follows in this case.

Case b. Let the two consecutive points of $F$ be $a_{k}, a_{r}^{\prime}$, a zero of $S^{\prime}(x)$. For convenience, suppose that $a_{k}<x_{r}^{\prime}$. (In case $a_{k}=x_{r}^{\prime}$, then it can be shown that $V(x)$ has also a zero at this $a_{k}[\mathbf{3 b}]$.) Then again, 
$G(x)$ decreases continuously from $+\infty$ to $-\infty$ as $x$ varies from $a_{k}$ to $x_{r}^{\prime}$. Thus the interval $\left(a_{k}, x_{r}^{\prime}\right)$ contains a point of $E$, namely the zero of $G$ in $\left(a_{k}, x_{r}^{\prime}\right)$.

Case c. Suppose the two consecutive points of $F$ are $x_{k}^{\prime}$ and $x_{k+1}^{\prime}$, both zeros of $S^{\prime}(x)$. Then, since the zeros of $S(x)$ are all real and simple [3a], $S(x)$ has one zero in $\left(x_{k}^{\prime}, x_{k+1}^{\prime}\right)$ and this completes the proof.

It may be noted that an $a_{j}$ and a zero $x_{l}^{\prime}$ of $S^{\prime}(x)$ may coincide. We have shown that this is possible if and only if this $a_{j}$ is also a zero of $V(x)$ [3b]. We show now that no two points of $E$ can coincide. It is known that zeros of $S(x)$ are simple and those of $V(x)$ are also distinct. It then suffices to prove the following

TheORem 3. No zero of a Van Vleck polynomial $V(x)$ is a zero of the corresponding Stieltjes polynomial $S(x)$.

Proof. If possible, let $x_{k}$ be a zero of both the polynomials $V(x)$ and $S(x)$. Let $S(x)=\left(x-x_{k}\right) T(x)$, then $T\left(x_{k}\right) \neq 0$ and $T(x)$ has all its zeros distinct. Also,

$$
\begin{gathered}
S^{\prime}(x)=\left(x-x_{k}\right) T^{\prime}(x)+T(x), \quad S^{\prime \prime}(x)=\left(x-x_{k}\right) T^{\prime \prime}(x)+2 T^{\prime}(x), \\
S^{\prime \prime \prime}(x)=\left(x-x_{k}\right) T^{\prime \prime \prime}(x)+3 T^{\prime \prime}(x) .
\end{gathered}
$$

Thus,

$$
S^{\prime}\left(x_{k}\right)=T\left(x_{k}\right) \neq 0, \quad S^{\prime \prime}\left(x_{k}\right)=2 T^{\prime}\left(x_{k}\right), \quad S^{\prime \prime \prime}\left(x_{k}\right)=3 T^{\prime \prime}\left(x_{k}\right) .
$$

Let $V(x)=\left(x-x_{k}\right) R(x)$. Then, equation (1.1) becomes,

$$
S^{\prime \prime}(x)+\left(\sum_{j=1}^{p} \frac{\alpha_{j}}{x-a_{j}}\right) S^{\prime}(x)=-\frac{\left(x-x_{k}\right)^{2} T(x) R(x)}{\prod_{j=1}^{p}\left(x-a_{j}\right)} .
$$

Equation (2.2) shows that the function

$$
F(x) \equiv S^{\prime \prime}(x)+\left(\sum_{j=1}^{p} \frac{\alpha_{j}}{x-a_{j}}\right) S^{\prime}(x)
$$

has a double zero at $x_{k}$. Thus, $F\left(x_{k}\right)=F^{\prime}\left(x_{k}\right)=0$. Let

$$
T(x)=\prod_{j=1 ; j \neq \boldsymbol{k}}^{n}\left(x-x_{j}\right),
$$

then $x_{j}$ are real, distinct and $x_{j} \neq x_{k},(j=1, \cdots, n ; j \neq k)$. Hence,

$$
\frac{T^{\prime}\left(x_{k}\right)}{T\left(x_{k}\right)}=\sum_{j=1 ; j \neq k}^{n} \frac{1}{x_{k}-x_{j}}
$$

and 
Also,

$$
\begin{aligned}
\frac{T^{\prime \prime}\left(x_{k}\right)}{T\left(x_{k}\right)} & =\sum_{r<j=1 ; j, r \neq k}^{n} \frac{2}{\left(x_{k}-x_{j}\right)\left(x_{k}-x_{r}\right)} \\
& =\left[\sum_{j=1 ; j \neq k}^{n} \frac{1}{x_{k}-x_{j}}\right]^{2}-\sum_{j=1 ; j \neq k}^{n} \frac{1}{\left(x_{k}-x_{j}\right)^{2}} .
\end{aligned}
$$

$$
\frac{S^{\prime \prime}\left(x_{k}\right)}{S^{\prime}\left(x_{k}\right)}=\frac{2 T^{\prime}\left(x_{k}\right)}{T\left(x_{k}\right)}=2 \sum_{j=1 ; j \neq k}^{n} \frac{1}{x_{k}-x_{j}} .
$$

Now, $F\left(x_{k}\right)=0$ gives

or,

$$
2 \sum_{j=1 ; j \neq k}^{n} \frac{1}{x_{k}-x_{j}}+\sum_{j=1}^{p} \frac{\alpha_{j}}{x_{k}-a_{j}}=0
$$

$$
2 \sum_{j=1 ; j \neq k}^{n} \frac{1}{x_{k}-x_{j}}=-\sum_{j=1}^{p} \frac{\alpha_{j}}{x_{k}-a_{j}} .
$$

Also, $F^{\prime}\left(x_{k}\right)=0$ gives,

$$
3 T^{\prime \prime}\left(x_{k}\right)+2\left(\sum_{j=1}^{p} \frac{\alpha_{j}}{x_{k}-a_{j}}\right) T^{\prime}\left(x_{k}\right)-\left(\sum_{j=1}^{p} \frac{\alpha_{j}}{\left(x_{k}-a_{j}\right)^{2}}\right) T\left(x_{k}\right)=0,
$$

or, since $T\left(x_{k}\right) \neq 0$,

$$
3 \frac{T^{\prime \prime}\left(x_{k}\right)}{T\left(x_{k}\right)}+2\left(\sum_{j=1}^{p} \frac{\alpha_{j}}{x_{k}-a_{j}}\right) \frac{T^{\prime}\left(x_{k}\right)}{T\left(x_{k}\right)}-\sum_{j=1}^{p} \frac{\alpha_{j}}{\left(x_{k}-a_{j}\right)^{2}}=0 .
$$

Using equations (2.3) and (2.4), this reduces to

$$
\begin{aligned}
3\left[\sum_{j=1 ; j \neq k}^{n} \frac{1}{x_{k}-x_{j}}\right]^{2} & -3 \sum_{j=1 ; j \neq k}^{n} \frac{1}{\left(x_{k}-x_{j}\right)^{2}} \\
& +2\left(\sum_{j=1}^{p} \frac{\alpha_{j}}{x_{k}-a_{j}}\right)\left(\sum_{j=1 ; j \neq k}^{n} \frac{1}{x_{k}-x_{j}}\right) \\
& -\sum_{j=1}^{p} \frac{\alpha_{j}}{\left(x_{k}-a_{j}\right)^{2}}=0 .
\end{aligned}
$$

In view of equation (2.5), this can be written as

$$
\begin{aligned}
-3 \sum_{j=1 ; j \neq k}^{n} \frac{1}{\left(x_{k}-x_{j}\right)^{2}} & -\left[\sum_{j=1 ; j \neq k}^{n} \frac{1}{x_{k}-x_{j}}\right]^{2} \\
& -\sum_{j=1}^{p} \frac{\alpha_{j}}{\left(x_{k}-a_{j}\right)^{2}}=0 .
\end{aligned}
$$


In the left-hand side of equation (2.6), each term is negative and hence the sum cannot vanish. This contradiction leads to the desired result.

3. Proof of Theorem 1. Let the $k$ consecutive intervals $\left(a_{j}, a_{j+1}\right)$, $\cdots,\left(a_{j+k-1}, a_{j+k}\right)$ contain $r(1 \leqq r \leqq n)$ zeros of $S(x)$. Let these zeros be $x_{m+1}<\cdots<x_{m+r}$. Then the following situations arise:

Case I. The intervals $\left(a_{j}, x_{m+1}\right)$ and $\left(x_{m+r}, a_{j+k}\right)$ contain each one zero of $S^{\prime}(x)$. In this case the interval $\left(a_{j}, a_{j+k}\right)$ contains precisely $(r+1)$ zeros of $S^{\prime}(x)$. This interval, therefore, contains $(k+r+2)$ points of the set $F$. By Theorem 2, therefore, there are exactly $(k+r+1)$ points of $E$ in these $k$ intervals. Since we have supposed that these intervals contain $r$ zeros of $S(x)$, it follows that the intervals $\left(a_{j}, a_{j+1}\right), \cdots,\left(a_{j+k-1}, a_{j+k}\right)$ contain exactly $(k+1)$ zeros of $V(x)$.

Case II. The interval $\left(a_{j}, x_{m+1}\right)$ contains one zero of $S^{\prime}(x)$ and $\left(x_{m+r}, a_{j+k}\right)$ does not contain any zero of $S^{\prime}(x)$. In such a case, $S^{\prime}(x)$ has $r$ zeros in $\left(a_{j}, a_{j+k}\right)$ and hence $\left(a_{j}, a_{j+k}\right)$ contains $(k+r+1)$ points of $F$. By Theorem 2, there are exactly $(k+r)$ points of $E$. Thus $\left(a_{j}, a_{j+k}\right)$ contains $k$ zeros of $V(x)$.

Case III. Neither of the intervals $\left(a_{j}, x_{m+1}\right)$ and $\left(x_{m+r}, a_{j+k}\right)$ contains any zero of $S^{\prime}(x)$. Here $\left(a_{j}, a_{j+k}\right)$ contains $(r-1)$ zeros of $S^{\prime}(x)$ and hence $(k+r)$ points of $F$. By Theorem $2,\left(a_{j}, a_{j+k}\right)$ contains exactly $(k+r-1)$ points of $E$ and hence $(k-1)$ zeros of $V(x)$.

In case $j=1$, the situation of Case I cannot prevail. For, in this case, $\left(a_{1}, x_{m}\right)$ does not contain any zero of $S^{\prime}(x)$, because $x_{m}$ is the smallest zero of $S(x)$ in $\left(a_{1}, a_{k+1}\right)$. Also, if $j=p-k$, the Case I is not possible, because $\left(x_{m}, a_{p}\right)$ does not contain any zero of $S^{\prime}(x)$. In this case, $x_{m}$ is the largest zero of $S(x)$.

Finally we consider the case when the $k$ intervals $\left(a_{j}, a_{j+1}\right), \cdots$, $\left(a_{j+k-1}, a_{j+k}\right)$ do not contain any zero of $S(x)$. In such a situation the interval $\left(a_{j}, a_{j+k}\right)$ contains at most one zero of $S^{\prime}(x)$ and hence at most $(k+2)$ points of $F$. By Theorem 2, therefore, $\left(a_{j}, a_{j+k}\right)$ contains at most $(k+1)$ points of $E$, or equivalently, at most $(k+1)$ zeros of $V(x)$. It may be noted, however, that in this case $V(x)$ will have at least $k$ zeros in $\left(a_{j}, a_{j+k}\right)$, and that being possible only when $S^{\prime}(x)$ has no zero in $\left(a_{j}, a_{j+k}\right)$. It is easy to see that when $j=1$ or $j=p-k, S^{\prime}(x)$ has no zero in $\left(a_{j}, a_{j+k}\right)$ and hence $V(x)$ has precisely $k$ zeros in this interval in such a case. The proof of Theorem 1 is now complete.

If we set in Theorem $1, k=p-1$ and $j=1$ and note that each $V(x)$ is a polynomial of degree $(p-2)$ we have the following result due to Van Vleck [5].

Theorem (Van Vleck). All the zeros of $V(x)$ lie in $\left(a_{1}, a_{p}\right)$. 


\section{BIBLIOGRAPHY}

1. M. Bôcher, The roots of polynomials which satisfy certain linear differential equations of the second order, Bull. Amer. Math. Soc. 4 (1898), 256-258.

2. M. Marden, Geometry of polynomials, 2nd ed., Math. Surveys No. 3, Amer. Math. Soc., Providence, R.I., 1966.

3a. G. M. Shah, Zeros of polynomial solutions of generalized Lamé differential equation, Doctoral dissertation, Univ. of Wisconsin-Milwaukee, 1966.

3b. - On the zeros of Stieltjes and Van Vleck polynomials, Illinois J. Math. (to appear).

4. T. J. Stieltjes, Sur certains polynômes que verifient une équation différentielle linéaire du second ordre et sur la théorie des fonctions de Lamé, Acta Math. 6 (1885), 321-326.

5. E. B. Van Vleck, On the polynomials of Stieltjes, Bull. Amer. Math. Soc. 4 (1898), 426-438.

University of Wisconsin-MilwaUkeE 